\title{
A Simplified Cellular Automaton Model for City Traffic
}

\section{DRAFT June 18, 2021}

\author{
P. M. Simon ${ }^{* \dagger}$ and K. Nagel ${ }^{* \dagger}$ \\ Los Alamos National Laboratory, TSA-DO/SA, MS-M997, Los Alamos, NM, 87545, USA ${ }^{\dagger}$, \\ Santa Fe Institute, 1399 Hyde Park Rd, Santa Fe NM 87501, USA *. (simonp,kai)@lanl.gov
}

We systematically investigate the effect of blockage sites in a cellular automaton model for traffic flow. Different scheduling schemes for the blockage sites are considered. None of them returns a linear relationship between the fraction of "green" time and the throughput. We use this information for a fast implementation of traffic in Dallas.

\section{INTRODUCTION}

In today's crowded world, space and money to build transportation systems which can fulfill all demand is often not available, or it is not desired to spend it on transportation system infrastructure. The result is congestion: from congested urban centers to congested innercity corridors to congested railways and congested airports. In consequence, some "forecasting" tool would be desirable. Unfortunately, congestion has the side effect that causal relations become much more spread both spatially and temporally [1]. If a road is crowded, the person may attempt a different route or a different mode (spatial spreading), or she may attempt the trip at a different time (temporal spreading) or even totally drop the trip. The result is that planning tools need to consider a much wider spatial and temporal context than ever before. Conceptually this means that for such problems the method needs to be "activity based", i.e. one needs to consider the whole process how people plan transportation in a daily or better weekly context (see, e.g., [2]).

Another effect of being in the congested regime is that one needs to worry a lot more than before about having a dynamically correct representation of the transportation system: For example, a peak-period spreading of traffic will not show up if one only models a 24-hour average situation (as many traditional tools do). Thus, we suddenly are faced with a problem where we need to introduce more dynamical correctness into the modeling while at the same time considering much wider temporal and spatial scales than before.

It is fairly obvious that, when faced with a dynamical problem, a "microscopic" approach, i.e. starting with a description of the smallest particles, is in terms of methodology the cleanest one. In transportation science, this currently means to consider individual travelers rather than, say, aggregated link flows. For example, it is difficult to include individual route choice behavior into a non-microscopic simulation. There is also some agree- ment that the currently most straightforward method to deal with microscopic approaches in complicated realworld contexts is computer simulation, as opposed to analytical techniques. Now, when faced with a computeintense problem, such as systematic scenario evaluations (see, e.g., 3 5]), or the simulation of the whole national transportation system [6], a very detailed and realistic microsimulation (see, e.g., [7, 8]) may be computationally too slow, or too data-intensive to run.

Alternatives here are simplified models which still capture the essentials of the dynamics at the transition to the congested regime. Since traffic in general is dominated by the bottlenecks in the system, these simulations concentrate on exactly these bottlenecks. The most important bottlenecks in urban systems are traffic lights. The natural outcome of this way of thinking are queuing-type models [9, 10]. For vehicles that enter the link, one calculates when they could arrive at the end of the link. When that time is reached in the simulation, they are added to a queue at the end of the link. They leave the queue once they have advanced to its beginning. The queue may have a limited service rate, which models capacity restrictions.

This paper approaches this problem from a slightly different angle. We use a very simple single-lane microsimulation to capture at least some of the dynamics that is going on on the link itself. This paper will provide a systematic approach to such a model. Sec. 2 will describe our model, the way capacity restrictions are modeled, what their behavior is, and what that means for the relation between the simulation and reality. In fact, capacity restrictions are simply modeled by "impurity sites" or temporary "blockages" (e.g. [11). Sec. 3 discusses an implementation and some results for a Dallas study. This is followed by a short discussion, highlighting the differences between our approach and other "queuing-type" approaches (Sec. 4), and a summary.

\section{A SIMPLIFIED APPROACH}

We present a simple simulation model of city traffic, using a combination of stochastic cellular automata (CA) and probabilistic transitions between streets. To represent the city network, we use the usual definition (e.g. [12]) for links and nodes: a link is a directed street segment, such as a bi-directional road divided into two links, whereas a node is an intersection; a link can also be 
defined by an input node and an output node. Vehicles are moved on a simple single-lane CA link, and are transferred from link to link following a simple probabilistic law based on the link's capacity.

\section{A. Links}

Links have different characteristics including length, speed-limit, number of lanes, maximum capacity, etc. The length is necessary to adjust the number of sites needed for the discrete approach of the CA. We use the standard reference of 7.5 meters for the length of one site [12,8, 13. Each site can be empty, or occupied by a vehicle with an integer velocity $v \in\left\{0 \ldots v_{\max }\right\}$. $v_{\max }=5$ gives good agreement with physical experiments.

Since each link is considered as a one-lane segment, vehicles are moved using the Nagel/Schreckenberg CA rule 13. Summarizing the one-lane CA model, the variable gap gives the number of unoccupied sites in front of a vehicle. $p_{\text {noise }}$ is the probability to randomly be slower than you could, and rand is a random number between 0 and 1. One iteration consists of the following three sequential steps which are applied in parallel to all cars:

1. Acceleration of free vehicles: IF $\left(v<v_{\max }\right)$ THEN $v=v+1$

2. Slowing down due to other cars: IF $(v>$ gap $)$ THEN $v=g a p$

3. Stochastic driver behavior: IF $(v>0)$ AND ( rand $<p_{\text {noise }}$ ) THEN $v=v-1$

For each link, we introduce an intrinsic probabilistic transition, which is a function of the capacity (maximum throughput). The one-lane model is faster and easier to implement compared to the multi-lane CA.

\section{B. Probabilistic transitions}

We introduce various probabilistic models to differentiate the existing links within a city, from high capacity segments such as freeways to low capacity segments such as arterials. If we consider only one-lane links, the probabilistic transition is introduced to control the output flow of a link. A high capacity link will produce a high output flow, while a low capacity link will produce a low output flow.

\section{Random traffic light}

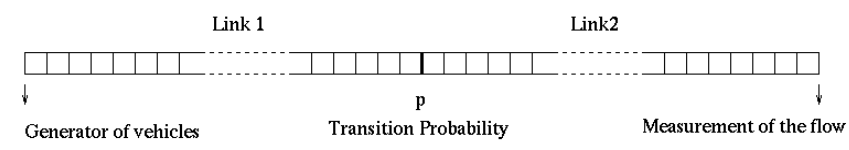

FIG. 1. Schema of the experiment

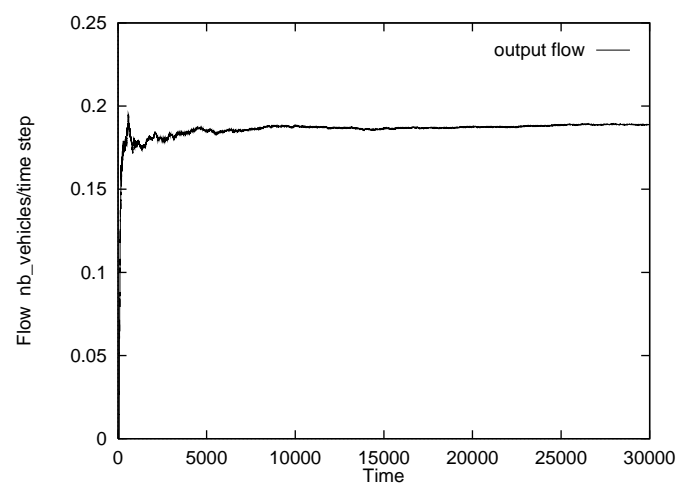

FIG. 2. Flow versus Time for a transition probability of 0.5 


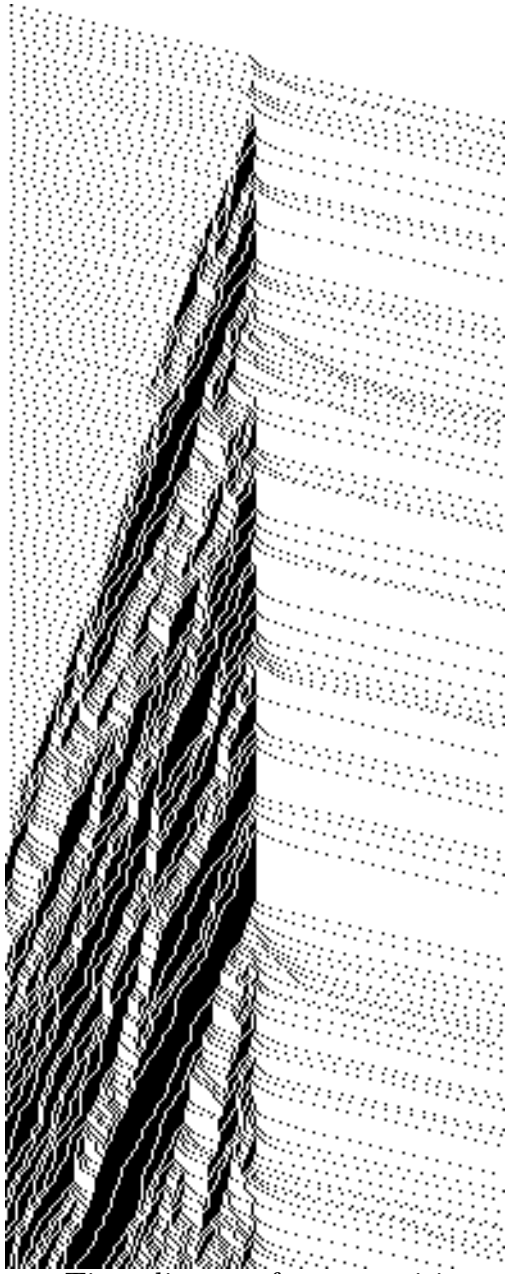

FIG. 3. Space-Time diagram for a transition probability of 0.5

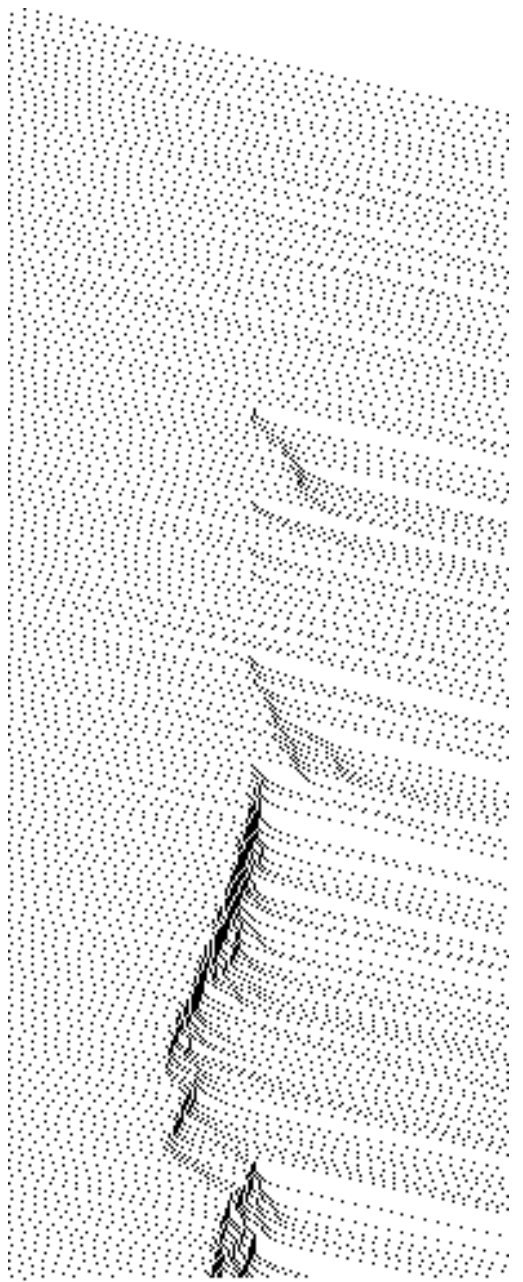

FIG. 4. Space time diagram for $\mathrm{p}=0.9$ 


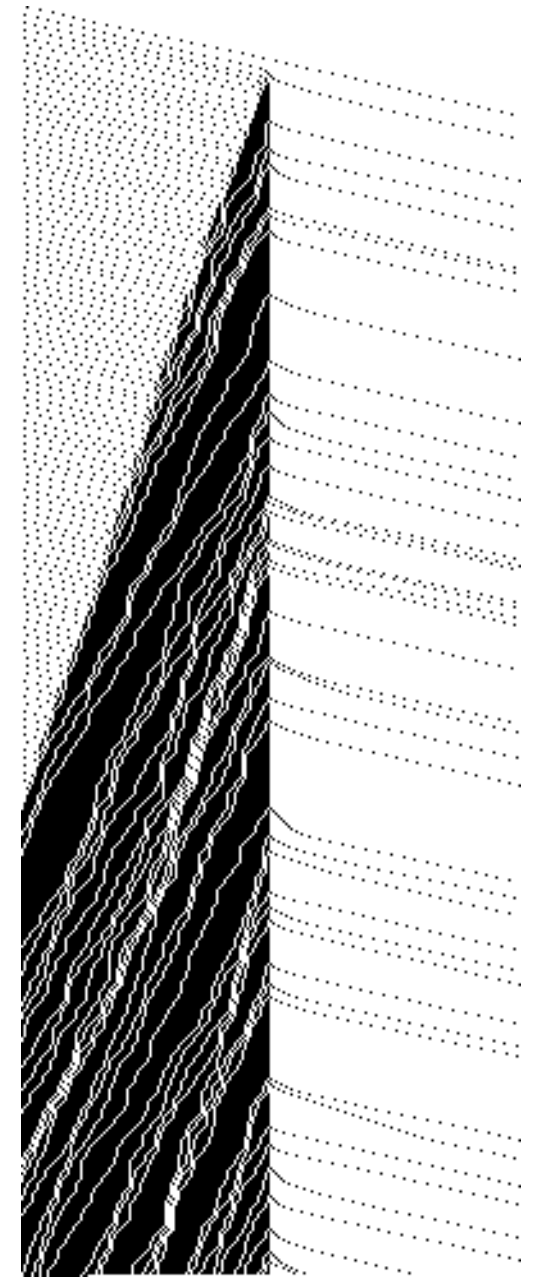

FIG. 5. Space time diagram for $\mathrm{p}=0.2$

Let us consider the experiment in fig [1], consisting of two consecutive links separated by a probabilistic transition $p_{\text {trans }}$. The first site of link 1 operates as a generator of vehicles, where one vehicle is introduced per $n$ iteration(s). The flow measured at the end of the second link versus the number of iterations is shown in fig 2]. The probabilistic transition is set to 0.5 in this example. The flow measured at iteration $t$ is the number of vehicles that left the second link until that moment, divided by $t$. As a result, the unity of the flow is vehicle per iteration. We introduce 1 vehicle every 3 iterations at the first site of the first link with maximum velocity 5 . This is enough to assure that the first link will reach around $1200 \mathrm{veh} / \mathrm{h}$ for a $p_{\text {noise }}$ of 0.5 , which is close to the maximum throughput of such a link in the CA implementation [13]. If the first site is not empty at the introducing time step, we do not add vehicles. The vehicle's velocities are updated by the one-lane CA model before reaching the intersection. If the vehicle is allowed to go through the intersection by the CA forward rule, we check the probabilistic transition.

If the generated random number is lower than the probability $p_{\text {trans }}$, the vehicle keeps its velocity and reaches the second link. In contrast, if the random number is greater than $p_{\text {trans }}$, we place a fictitious car in the first site of the second link in order to force the vehicle to brake and stop at the intersection. Technically: If a car reaches the last five sites of a link, it produces a random number. We introduce the simple algorithm:

1. Transition check:

IF (rand $<p_{\text {trans }}$ ) THEN normal CA-update ELSE gap=distance from the vehicle to the intersection

This situation is in principle well understood. The "impurity site" will create a reduced flow that can pass that site, and since flow needs to be conserved along the link, this sets the maximum throughput for the link [11, 14 17. Yet, in the context of the stochastic traffic cellular automaton as used here, we are only aware of Ref. [18], and the specific mechanism used there is not the one we wanted to use.

Figs [3] demonstrate the formation of traffic jams spreading to the beginning of the link, caused by braking of vehicles. The beginning of the second link can again be considered as a generator of vehicles. Nevertheless, the input flow and $p$ are not proportional.

To illustrate this comment, we conduct the same experiment with probabilistic transitions ranging from 0 to 1. The average flow obtained for each experiment is presented in fig [6]. For each data point, the flow is averaged in the time period $(5000,20000)$. See fig[2]. The intersection does not function as a perfect generator of service rate $p$. If a vehicle leaves the last site of the first link, this vehicle is not automatically replaced, due to the stochastic third step included in the one-lane CA model. This plot can be divided into three different parts:

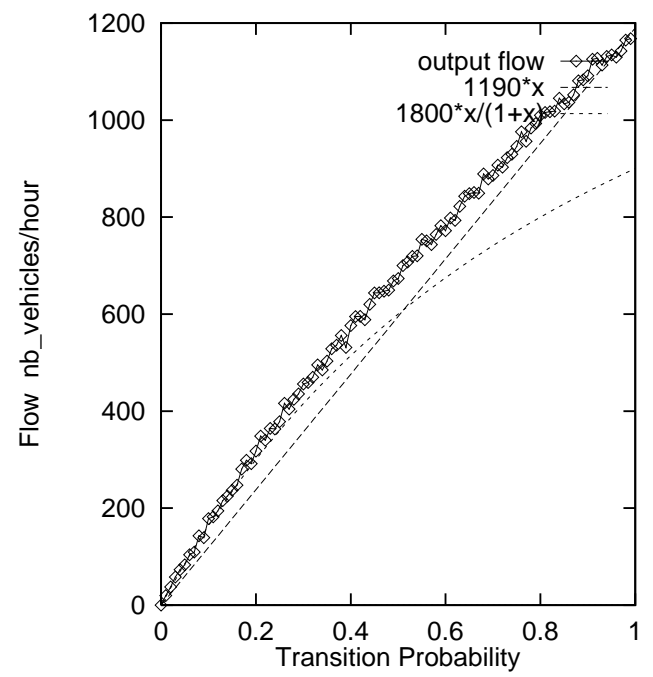

FIG. 6. Flow versus transition probabilities

(i) A high probabilistic transition ( $p_{\text {trans }}$ between 0.8 and 1.0) gives linear results with input flow. In this scenario, vehicles do not stop often at the intersection, thus 
the intersection does not work like a stop and start point. See fig[4].

(ii) A low probabilistic transition (between 0 and 0.4 ) gives results that can be explained by a simple hypothesis. Most of the cars stop at the intersection and form a compact traffic jam, as shown in fig[5]. There are no important spaces in this queue. Assuming that the second last site of the first link is always crowded, how many iterations does a vehicle need to go through the intersection? If a vehicle is on the last site of link 1, the vehicle needs $1 / p_{\text {noise }}$ iterations on average to advance, and then multiplied by $1 / p_{\text {trans }}$ to go through the intersection. Viewed from the perspective of the next following vehicle, that one needs to wait $1 / p_{\text {noise }} \cdot 1 / p_{\text {trans }}$ steps until the vehicle ahead is gone, and then another $1 / p_{\text {noise }}$ steps to move itself to arrive at the last site. As a result the average number of iterations for a vehicle to advance from the second last site of link 1, to the intersection is $1 / p_{\text {noise }}+1 /\left(p_{\text {noise }} p_{\text {trans }}\right)$. This could in theory be continued, but it would not necessarily get better because one would need to include the influence of "holes" in the queue; or, more technically: The approximation is only valid for $p_{\text {trans }} \rightarrow 0$, and second order corrections are thus negligible. In any case, the corresponding flow is $F \approx p_{\text {noise }} p_{\text {trans }} /\left(1+p_{\text {trans }}\right)$.

The function, $F$, shown in fig[6] fits well to the data measured for low values of $p$, while for $p \geq 0.4$ the hypothesis is no longer valid.

(iii) Figure [3] demonstrates what happens for probabilistic transitions between 0.4 and 0.8 at a microscopic level. Within the queue, holes are generated by the intersection and an analytical approach becomes more difficult. Periodically, vehicles pass through the intersection without braking and stopping, which produces a higher flow compared to the linear relationship illustrated in fig 6 .

Many experiments can be conducted using other probability distributions for the intersection. The model previously described operates like a random traffic light, where the light becomes green with the probability $p_{\text {trans }}$, which is also the fraction of the time the light is green: $f_{\text {green }}=p_{\text {trans }}$. This model can be considered to be one between two extreme distributions, where in between the extreme cases one can encounter an infinite number of distributions that keep the fraction of a green light of the total time of a traffic cycle constant. The first distribution is a classical traffic light. The green fraction here is straightforward: $f_{\text {green }}=T_{\text {green }} /\left(T_{\text {green }}+T_{\text {red }}\right)$. We call the second model a Dirac traffic light. As we work with discrete systems, the objective is to set a green light or a red light on only one time unit, equally spaced on a cycle. The green fraction is $f_{\text {green }}=1 /\left(1+T_{\text {red }}\right)$ for $T_{\text {red }} \geq 1$ (and $T_{\text {green }}=1$ by definition) or $1-1 /\left(1+T_{\text {green }}\right)$ for $T_{\text {green }} \geq 1$. All three distributions are illustrated in fig [7].
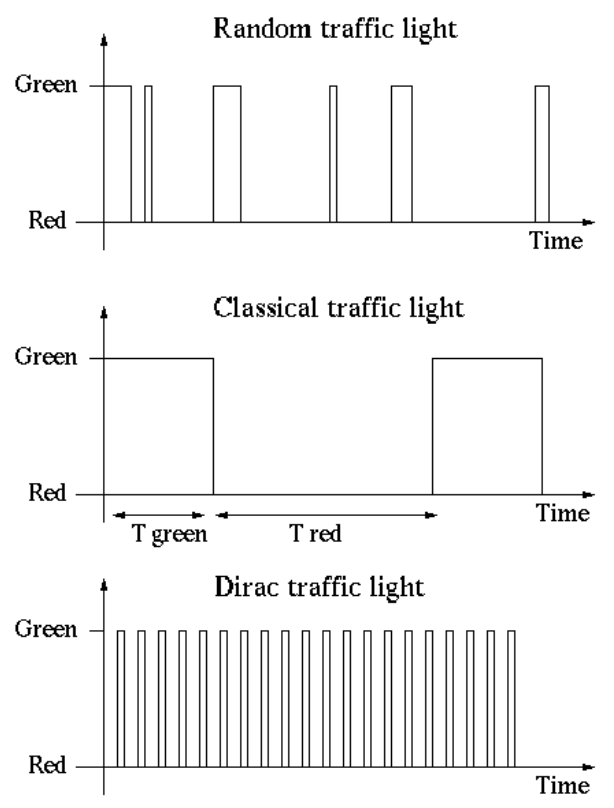

FIG. 7. Different distribution probabilities

Next, we present the same experiments discussed above, for these two distributions.

\section{Normal traffic light}

We repeat the same experiment described in fig[1] with a normal traffic light at the intersection. 


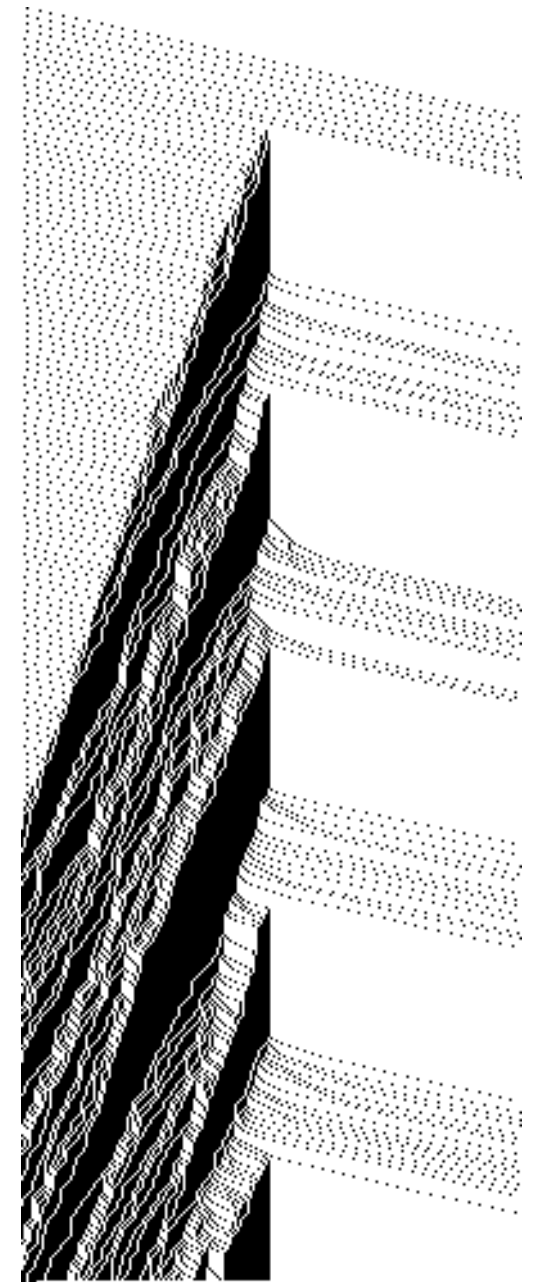

FIG. 8. Space-time diagram normal traffic light $\mathrm{p}=0.5$

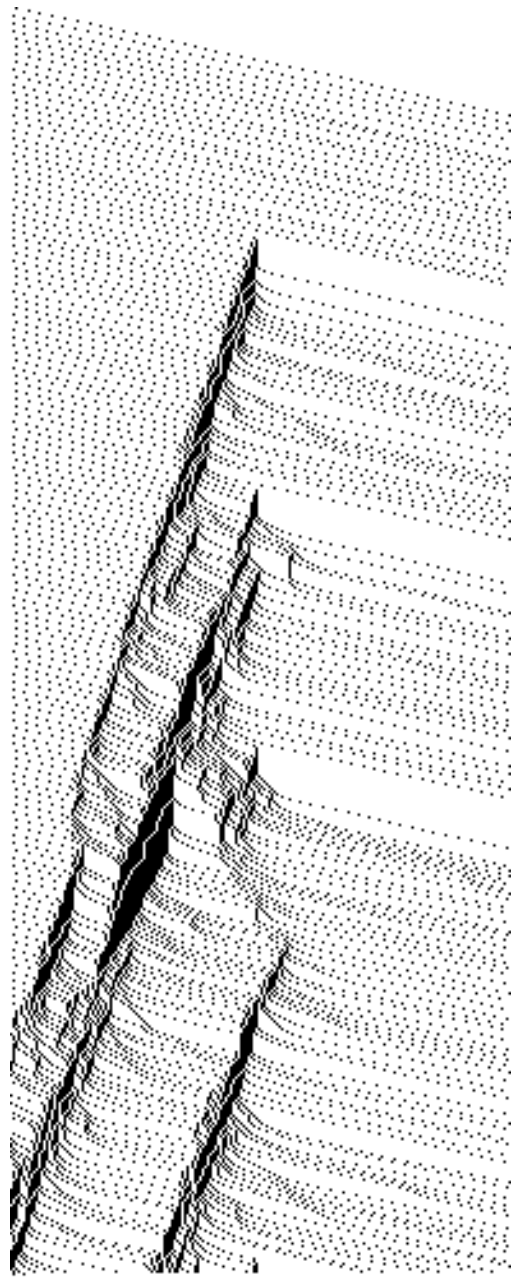

FIG. 9. Space-time diagram normal traffic light $\mathrm{p}=0.9$

The dissolution of a queue as the light turns periodically green is shown in fig [8]. This phenomenon does not provide an easy analytical solution. For each green fraction $f_{\text {green }}$ ranging from 0 to 1 , the input flow of the second link is measured and is illustrated in fig 10$]$. This relationship is almost linear. For high values of transitions, vehicles still have to stop occasionally, which decreases the output flow. Figure 9], when compared to the space-time diagram produced by the random traffic light fig $\mathbb{\text { ] }}$, displays a lack of fluidity. 


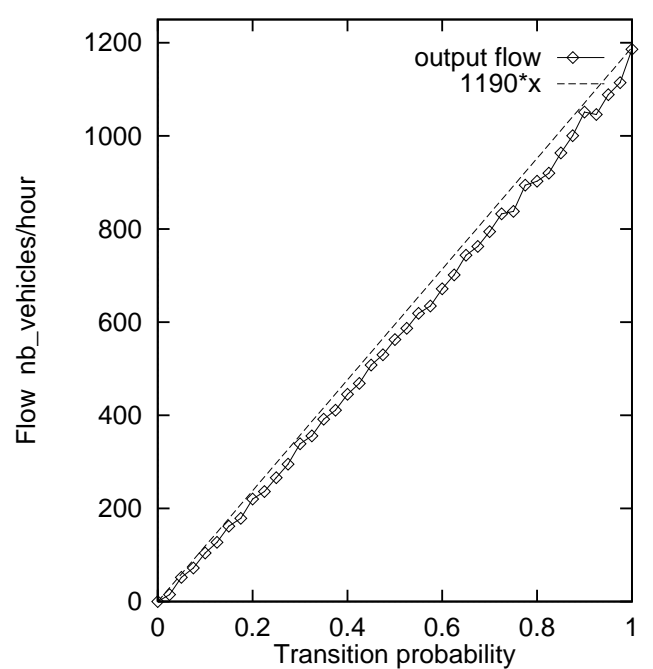

FIG. 10. Flow versus Transition probability

\section{Dirac traffic light}

The Dirac traffic light generates the highest flow for a given $f_{\text {green }}$ in the experiment or fig[1]. The space-time diagram performed with a probabilistic transition of 0.5 is given as an example. In this case, the traffic light is successively green and red. Figure 11] shows less compact traffic jams at the end of the first link than the other space-time diagrams for the same probabilistic transition. This is still due to the vehicles that pass through the intersection at maximum velocity without braking. The analytic explanation for this is the fact that the parallel update tends to generate states where particles are followed by holes, sometimes called "particle-hole attraction" 19.

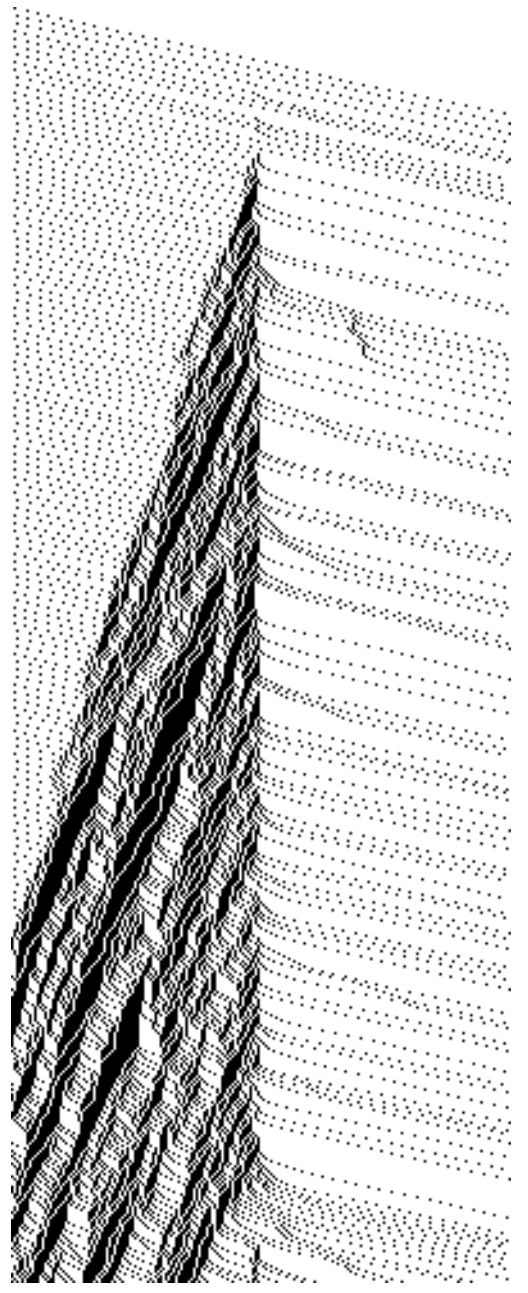

FIG. 11. Space-time diagram Dirac traffic light $\mathrm{p}=0.5$ 


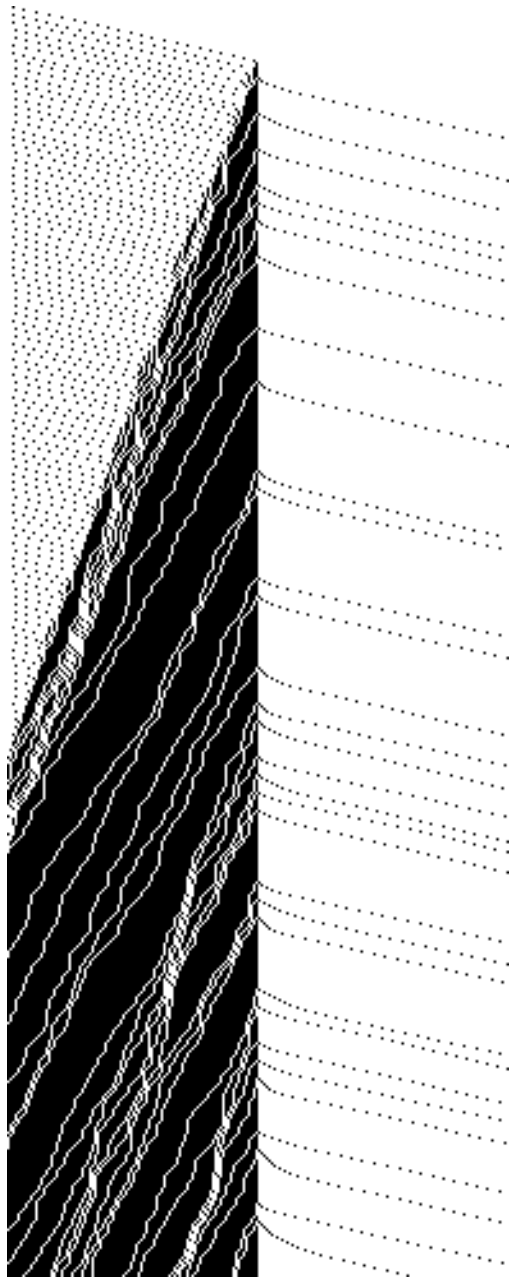

FIG. 12. Space-time diagram Dirac traffic light $\mathrm{p}=0.16$

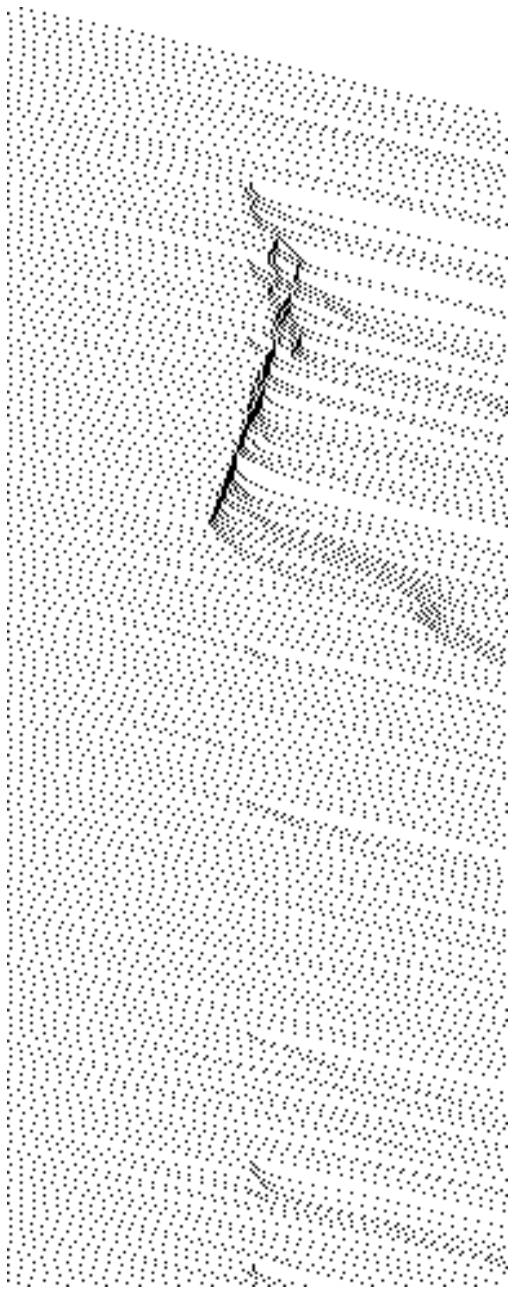

FIG. 13. Space-time diagram Dirac traffic light $p=0.9$

The output flow of link 1 for any value of $p$ is much higher than the two flows measured previously for the two other probability distributions. There is no linear relation at any position on this diagram. The space-time diagrams plotted for a $p=0.16$ and $p=0.9$ exhibit more fluidity for the output traffic fig[12, 13]. 


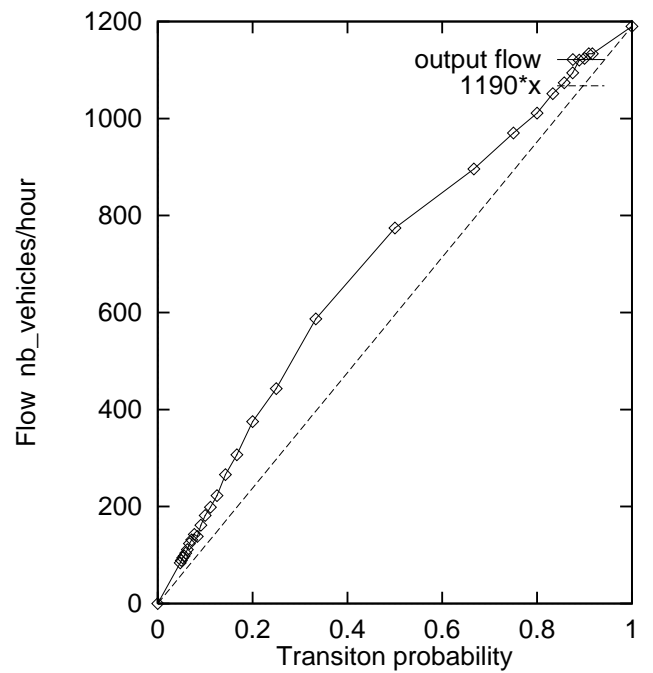

FIG. 14. Flow versus transition probability

\section{DALLAS}

\section{A. Implementation}

The normal traffic light model is the most linear model simulated in this paper. On the other hand, setting a traffic light at each intersection would cost computation time. The random traffic light presents the advantage to be checked only when a vehicle reaches the intersection. The vehicle generates a random number which allows it to drive trough the intersection or not.

We apply this model to the Dallas Fort-Worth area. The context is the so-called Dallas/Fort Worth case study 4,20] which has been done as part of the TRANSIMS (TRansportation ANalysis and SIMulation System) project [2]. TRANSIMS uses individual route plans for each individual traveler. A route plan consists of a starting time, a starting location, a list of links the vehicle intends to follow, and an ending location. A microsimulation in the TRANSIMS project such as the one described here is thus faced with the task to move these vehicles according to these specifications.

One immediately observes that one somehow has to correct for the fact that we are only using single-lane roads, i.e. our links will usually not be able to carry the prescribed number of vehicles. We solve that problem by using a sub-sample of the plans. The size of that subsample is obtained as follows:

- $p_{\text {noise }}=0.5$ results in a maximum throughput of a link of approximately $1200 \mathrm{veh} / \mathrm{h}$ (using $p_{\text {trans }}=$ $1)$.

- We search for the link with the highest capacity in the area we want to simulate. In our case, this was a four lane freeway with a capacity of $7800 \mathrm{veh} / \mathrm{h}$.

- We thus need to sub-sample the population by a factor of $1200 / 7800 \approx 0.154$, i.e. a route plan from the full plan-set is going to be used with a probability of 0.154 .

- Links which have a lower capacity than $7800 \mathrm{veh} / \mathrm{h}$ take this fact into account by using a value of $p_{\text {trans }}$ according to Fig. 6, i.e. if the value of the road is $C$, then the value $C \cdot 0.154$ is used on the $\mathrm{y}$-axis to find the correct value of $p_{\text {trans }}$ on the $\mathrm{x}$-axis.

A more precise calibration is more complicated than this because it also depends on the interplay between route planning and route execution. This is clearly out of the scope of this paper; further publications on the subject are in preparation.

\section{B. Simulation results}

In this section, we want to give some examples how this simulation is going to be used. These examples will be given in the context of the TRANSIMS Dallas/Fort Worth case study. That case study used as input a street network of the Dallas/Fort Worth area, containing 24662 links and 9864 nodes, and information of all trips in this area during a 24 hour period (approx. 10 million trips). The study focused on a busy 5 miles times 5 miles area north of downtown Dallas, and on the time between $5 \mathrm{am}$ and 10am. This still involved 300000 trips. As mentioned above, micro-simulations in the TRANSIMS project are route-plan driven. Thus, for each of these 300000 trips, route plans were calculated. The fact that drivers adjust to congestion caused by other drivers was taken into account by iteration several times between the route planning and the micro-simulation. For further information, see Refs. 2022].

One important specification missing in the above description of the micro-simulation is how vehicles enter and leave the simulation. TRANSIMS specifies parking locations along links, which represent all parking opportunities that can be reached from this link. In order to prevent that the traffic that leaves parking unrealistically disturbs the traffic flow, vehicles from the parking locations are only inserted if $v_{\max }$ sites backwards from the parking location are empty. If the space is not free, the car is placed in a queue, waiting to enter the simulation in one of the following iterations. 


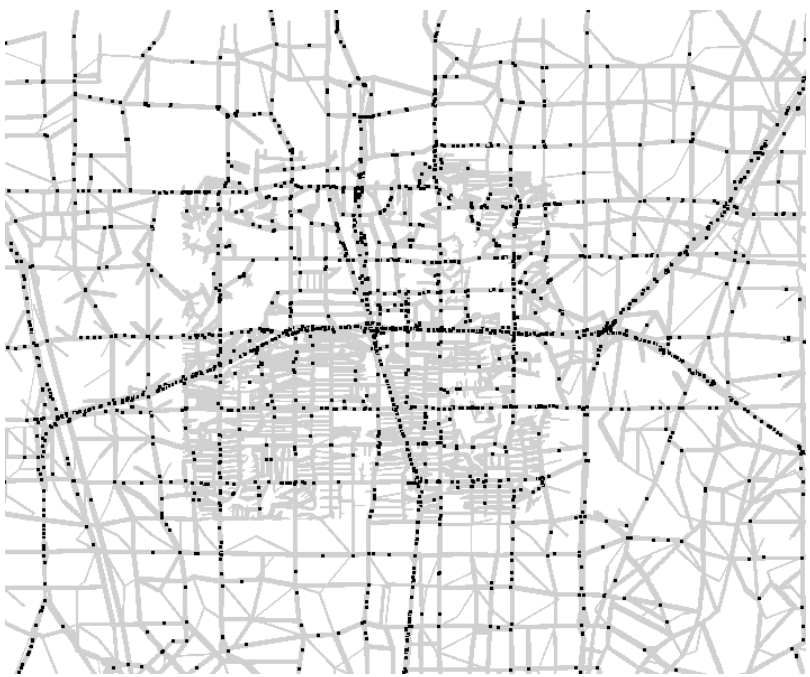

FIG. 15. Snapshot of the case study at 7:00

A snapshot of such a simulation with the model described in this paper can be found in Fig[15]. The denser square area in the center represents the study area, where all streets including local streets were represented in the data base. For this example, also the streets outside that area were simulated. Dots denote individual vehicles. In this plot, most of the traffic is on the freeways, as is realistic. Also, one notes that for lower capacity road, traffic is mostly queued up towards the end, as one would expect from the dynamics of the model. Yet, this is really not too unrealistic since also in reality traffic through minor roads tends to queue up at the ends.

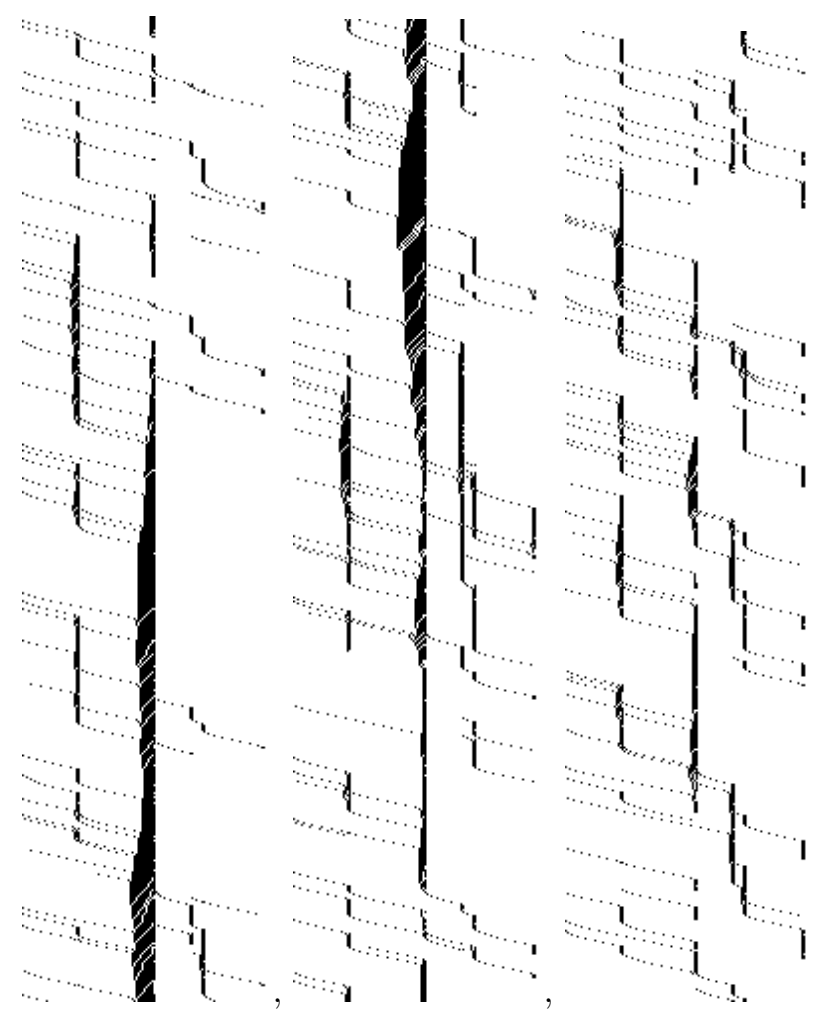

FIG. 16. Space-time plot of a particular link (Beltline Rd., an east-west arterial in the northern part of the area) from 7:00am to 7:05am (left), 7:30am to 7:35am (middle), and 8:00am to 8:05am (right.)

The space-time diagram of five consecutive links is shown in fig 16]. These links are a part of an east-west arterial located in the north of the study area. The figure shows nicely how queues built up at the end of links due to the capacity restrictions.

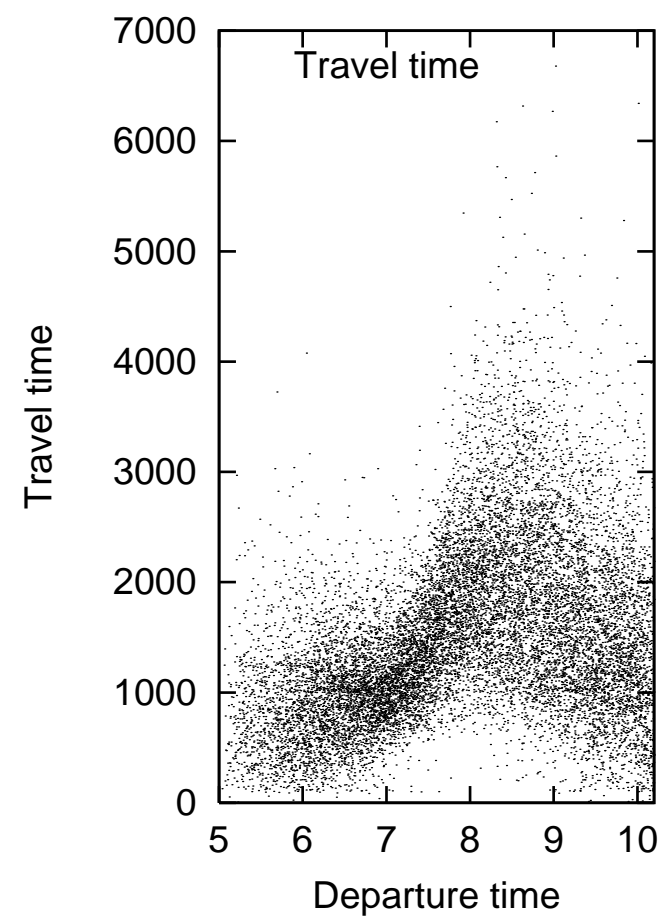

FIG. 17. Travel times versus departure time

Many statistics can be extracted from the simulation. As a further example, we present the travel time versus departure time for each vehicle (Fig. 17). This figure shows nicely that even such a simple simulation as the one described in this paper can, given a realistic trip demand input, display the higher travel times during the rush hour. 


\section{Computational performance}

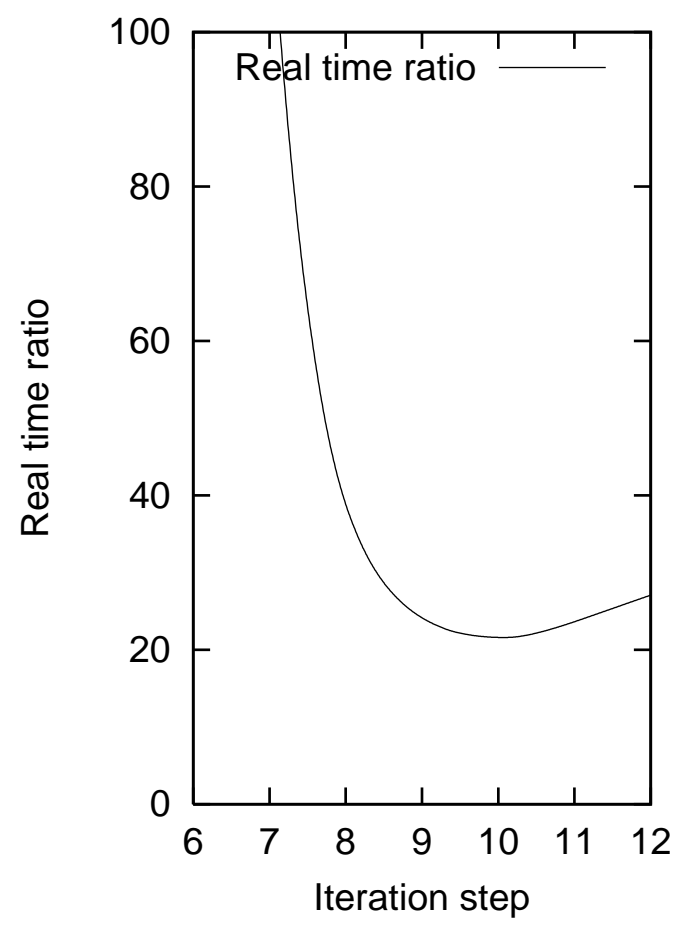

FIG. 18. Real time ratio

We present a performance diagram in fig 18$]$ where we introduce the RTR versus the simulation time. The RTR is the ratio of the real time on the simulation time. This example of simulation was executed on a SUN UltraSparc CPU with $250 \mathrm{MHz}$ where approximately 46000 plans were simulated in the whole Dallas Fort-Worth area. The diagram fig[18] shows a ratio of 23 in the middle of the rush period, but in average the ratio is around 28. This clearly shows that simulations like the one described here have enough computational speed for thorough investigations of traffic problems.

\section{DISCUSSION}

Transportation models using simplified link dynamics ultimately fail to generate some aspects of a complicated reality, such as a turn pocket having a queue spill-back into the lanes going straight. Yet, as pointed out in the introduction, using a highly realistic model sometimes is not an option, for example because of computational constraints or data collection constraints. In such cases, knowing the different limitations of the simplified models becomes crucial.

For example, the different simplified models handle queue discharge dynamics in different ways. In our model, when a vehicle is moved to the next link, this leaves a "hole" on the link where the vehicle comes from.
In the next time step, that hole may or may not be filled by an advancing vehicle, according to the stochastic driving rules. In a congested situation, this hole slowly travels backwards, until it eventually reaches the other end of the link, allowing a new vehicle to enter the link. In Ref. [10], holes are transmitted instantaneously to the other end of the link. The method of Ref. [9] assumes infinite queuing capacity on each link. It is clear that all three methods will generate different dynamics.

As another example, in fairly realistic models, sources and sinks for traffic are better located in the middle of links instead of at nodes. Vehicles attempt to squeeze in between other vehicles at that location on the link. If the link gets congested, the additional vehicles will have trouble finding additional space to squeeze in. It is clear that models who totally give up a representation of traffic dynamics on the link such as [10,9] will lead to different behavior for traffic sources and sinks.

Certainly, the simple queuing models could compensate for that. Yet, that usually comes at the price of being tedious. Often, it will be more straightforward to move directly to a higher fidelity (but usually computationally slower and more data intensive) micro-simulation. We believe that, at the current stage, it is more important to really understand the dynamical differences between different models and to compare their behavior in realworld applications, than to attempt to improve simplified models in non-intuitive ways.

Last but not least, the model presented in this paper actively moves vehicles along links with roughly realistic dynamics. This makes graphical output such as in Fig. 15 much more intuitive and appealing.

\section{SUMMARY}

"Blockage" sites, i.e. sites which move particles or vehicles only a fraction of the time, reduce the maximum throughput of a link of cellular automata models for traffic flow and particle movement studies. We have systematically tested the effects of three different blockage schemes, where one was the usual random draw, one was a regular traffic light with long red and green times, and one was what we called a "Dirac" traffic light because it had 1-second spikes of one color. In general, there is no linear relation between the fraction of green time and the throughput. The Dirac traffic light returned the highest throughput; the explanation for this is the "particlehole" attraction that can be found in the type of cellular automaton that was used. Since none of the timing schemes returns a totally linear relation, we used the random scheme in an implementation of traffic in Dallas. We showed some exemplary results of this implementation. 


\section{ACKNOWLEDGMENTS}

This work has been performed at Los Alamos National Laboratory, which is operated by the University of California for the U.S. Department of Energy under contract W-7405-ENG-36.
[19] M. Schreckenberg, A. Schadschneider, K. Nagel, and N. Ito, Phys. Rev. E 51, 2939 (1995).

[20] K. Nagel and C.L.Barrett, International Journal of Modern Physics C 8, 505 (1997).

[21] M. Rickert et al, in preparation.

[22] K. Nagel et al, in preparation.

[1] K. Nagel and S. Rasmussen, in Artificial Life IV: Proceedings of the Fourth International Workshop on the Synthesis and Simulation of Living Systems, edited by R. A. Brooks and P. Maes (MIT Press, Cambridge, MA, ADDRESS, 1994), pp. 222-235.

[2] TRANSIMS, TRansportation ANalysis and SIMulation System, Los Alamos National Laboratory, Los Alamos, U.S.A. See www-transims.tsasa.lanl.gov.

[3] M. Rickert, P. Wagner, and C. Gawron, Real-time traffic simulation of the German Autobahn Network, 1996, in press.

[4] R. Beckman et al, Los Alamos Unclassified Report LAUR to be released, Los Alamos National Laboratory, TSA-Division, Los Alamos NM 87545, USA (unpublished).

[5] H. Mahmassani, T. Hu, and R. Jayakrishnan, in Urban traffic networks: Dynamic flow modeling and control, edited by N. Gartner and G. Improta (Springer, Berlin, New York, 1995).

[6] D. Anson and R. Nelson, LA-UR 97-4389, Los Alamos National Laboratory, http://www.lanl.gov/ (unpublished).

[7] R. Wiedemann, Schriftenreihe Heft 8, Institute for Transportation Science, University of Karlsruhe, Karlsruhe, Germany (unpublished).

[8] K. Nagel et al., TRANSIMS traffic flow characteristics, submitted, also LA-UR 97-3530.

[9] H. Simão and W. Powell, Transportation Science 26, 296 (1992).

[10] C. Gawron, An iterative algorithm to determine the dynamic user equilibrium in a traffic simulation model, preprint.

[11] S. Janowsky and J. L. Lebowitz, Physical Review E 45, 618 (1992).

[12] M. Rickert and K. Nagel, International Journal of Modern Physics C 8, 483 (1997).

[13] K. Nagel and M. Schreckenberg, J. Phys. I France 2, 2221 (1992).

[14] S. Yukawa, M. Kikuchi, and S. Tadaki, J. Phys. Soc. Japan 63(10), 3609 (1994).

[15] Z. Csahók and T. Vicsek, J. Physics A: Mathematics and General 27, L591 (1994).

[16] L. Vilar and A. de Souza, Physica A 211(1), 84 (1994).

[17] K. H. Chung and P. M. Hui, J. Phys. Soc. Japan 63(12), 4338 (1994).

[18] H. Emmerich and E. Rank, Physica A 216(4), 435 (1995). 